\title{
Natural Resources for Human Health: A New Interdisciplinary Journal Dedicated to Natural Sciences
}

Natural science is a science that deals with the interpretation, prediction and understanding of a natural phenomenon. It is divided into life and physical sciences which are subdivided into physics, chemistry, earth sciences and astronomy. Numerous journals specifically catering to these disciplines are cited in Scopus and Web of Science (WoS. Currently, 26674 journals have been indexed in the Web of Science. Among those, 20932 journals have given authors the option to choose open access of their articles and 4672 journals are published under the DOAJ Gold open access (data retrieved from Web of Sciences on 01.08.2021). The data suggest that the scientific world and the publishing sector are gradually moving towards open access policy, where researchers have free unrestricted access to the scientific content. It is interesting to note that only 56 journals are listed in the multidisciplinary sciences. Keeping this in mind, Natural Resources for Human Health (NRFHH), an interdisciplinary journal dedicated to natural sciences, has been launched.

NRFHH is an international open access journal that deliver high quality scientific articles to readers from various natural sciences domains. The NRFHH covers a wide range of natural science disciplines. Such fields could include almost all aspects of works bearing on natural compounds of plants, algae, microbes and animals. Each manuscript should have a clear hypothesis. In addition, high-quality review articles are also considered for publication based on a policy of integration for the propagation of novel research ideas and initiatives. The NRFHH publishes high-quality manuscripts related (but not limited) to life science research including biology, biological chemistry, medicinal chemistry, pharmaceutical formulations, agricultural and food chemistry, applications of natural resources and traditional medicine. Among the subjects covered by NRFHH are: taxonomy, conservation, genomics approaches for the conservation of critically endangered species, chemistry of living things, toxicology, pharmacology, pharmacognosy, nutrition and health, therapeutics, bioactive compounds, detoxification, biological activities, formulations from natural products, analysis, quality controls, food technology, agricultural and food safety, food analysis, cosmetics, dietary supplements, nutraceuticals, bio-pharmaceutical science, waste and co-product valorization, folkloric medicine, ethnobotany and ethnopharmacology.

The NRFHH is published by the Visagaa Publishing House. The editorial board is led by highly cited researcher Prof. Jesus Simal-Gandara, together with many other internationally recognised researchers. The editorial board comprises 37 editors from 22 countries (Figure 1), which strengthens the academic impact of the journal.

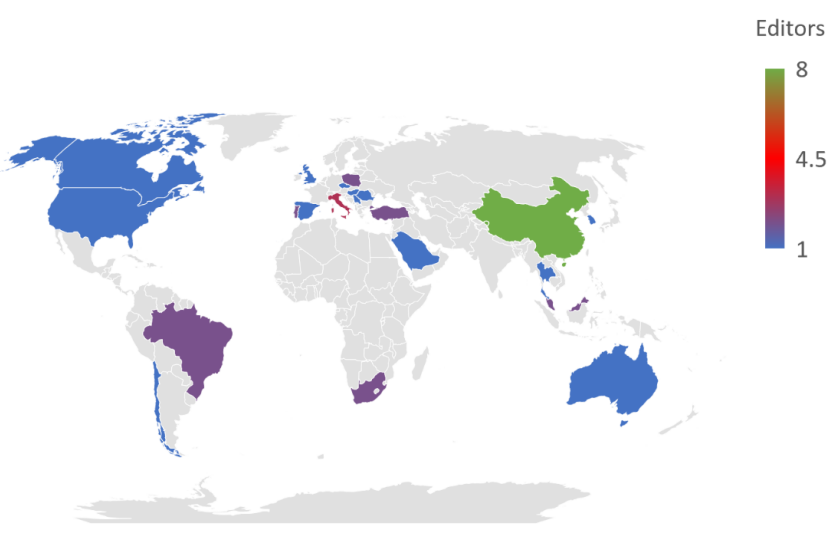

Figure 1. The geographical diversity of editorial board members of Natural Resources for Human Health.

In academic publishing, the role of expert reviewers is vital for safeguarding the merit and scientific quality of each submission and to support rapid publication within a given timeframe. The reviewers will be much appreciated by providing timely and constructive review reports. Without the prominent contributions of authors, reviewers and our valuable readers, the journal will not be successful. We want to establish a close scientific bond with you all. The journal already has many submissions. The journal already has many submissions. Enriching readers' access to high-quality scientific data is our mission at NRFHH.

\section{CONFLICT OF INTEREST}

The Authors disclose no conflicts of interest. Jesus Simal-Gandara, as Editor-in-Chief, and the other editorial board members had no involvement or access to information regarding this article's peer review. Kannan RR Rengasamy was solely responsible for the editorial process.

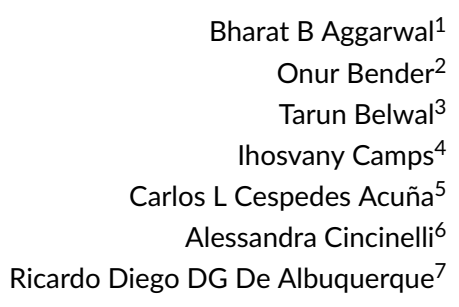

Bharat B Aggarwal ${ }^{1}$

Onur Bender ${ }^{2}$

Tarun Belwal ${ }^{3}$

arlos L Cespedes Acuña ${ }^{5}$

Alessandra Cincinelli6

Ricardo Diego DG De Albuquerque 


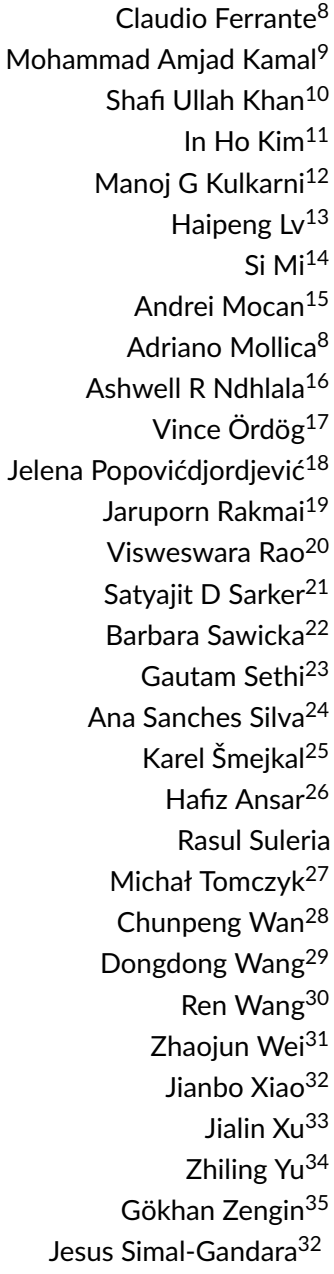

${ }^{1}$ Inflammation Research Center, San Diego, California; USA ${ }^{2}$ Biotechnology Institute, Ankara University, Ankara, Turkey

${ }^{3}$ Zhejiang Key Laboratory for Agri-Food Processing Zhejiang University, Hangzhou 310058, China

${ }^{4}$ Physics Department. Exact Science Institute, Federal University of Alfenas, UNIFAL-MG, Alfenas, Minas Gerais 37133-840, Brazil

${ }^{5}$ Basic Sciences Department, University of Bio Bio, Andres Bello Av. 720, 3800708, Chillan, Chile

${ }^{6}$ Department of Chemistry "Ugo Schiff", University of Florence, Italy

${ }^{7}$ Laboratory of Technology in Natural Products (LTPN - Universidade Federal Fluminense) Rua Miguel de Frias, 9 Icaraí, Niterói - RJ, Brazil ${ }^{8}$ Department of Pharmacy, University of "D'Annunzio" of Chieti-Pescara, 66100 , Italy

${ }^{9}$ King Fahd Medical Research Center, King Abdulaziz University, Saudi Arabia

${ }^{10}$ Department of Pharmacy, Abasyn University Peshawar, Khyber Pakhtunkhwa 25000, Pakistan

${ }^{11}$ Department of Animal Resource \& Science, Dankook University, Cheonan, 330-714 South Korea

${ }^{12}$ Research Centre for Plant Growth and Development, School of Life Sciences, University of KwaZulu-Natal Pietermaritzburg, South Africa

${ }^{13}$ Tea Research Institute, Chinese Academy of Agricultural Sciences, Hangzhou, China

${ }^{14}$ College of Food Science and Technology, Hebei Agricultural University, Baoding 071000, China
${ }^{15}$ Department of Pharmaceutical Botany, 'Iuliu Hațieganu' University of Medicine and Pharmacy, 23 Gheorghe Marinescu Street, 400337, Cluj-Napoca, Romania ${ }^{16}$ Green Biotechnologies Research Centre of Excellence, University of Limpopo, Sovenga 0727, South Africa

${ }^{17}$ Széchényi István University, Faculty of Agricultural and Food Science, Mosonmagyaróvár, Hungary

${ }^{18}$ University of Belgrade, Faculty of Agriculture, Belgrade, Serbia

${ }^{19}$ Kasetsart Agricultural and Agro-Industrial Product Improvement Institute (KAPI), Kasetsart University, Ngamwongwan Road, 10900 Bangkok, Thailand

${ }^{20}$ Department of Biomedical Sciences and Therapeutics, Faculty of Medicine and Health Sciences, Universiti Malaysia Sabah, Kota Kinabalu 88400, Sabah, Malaysia

${ }^{21}$ Centre for Natural Products Discovery (CNPD), School of Pharmacy and Biomolecular Sciences, Liverpool John Moores University, Byrom Street, Liverpool L3 3AF, UK ${ }^{22}$ Department of Plant Production Technology and Commodities Sciences, Faculty of Agrobioengineering, University of Life Sciences in Lublin, Poland

${ }^{23}$ Department of Pharmacology, Yong Loo Lin School of Medicine, National University of Singapore, 117600, Singapore

${ }^{24}$ National Institute of Agrarian and Veterinary Research, 4485-655 Vairão, Portugal

${ }^{25}$ Department of Natural Drugs, Faculty of Pharmacy, Masaryk University, 61200 Brno, Czech Republic ${ }^{26}$ School of Agriculture and Food, The University of Melbourne, Parkville, VIC 3010, Australia

${ }^{27}$ Department of Pharmacognosy, Faculty of Pharmacy with the Division of Laboratory Medicine, Medical University of Białystok, Ul. Mickiewicza 2a, 15-230, Białystok, Poland ${ }^{28}$ College of Agronomy, Jiangxi Agricultural University, Nanchang 330045, China

${ }^{29}$ Centre for Metabolism, Obesity, and Diabetes Research, Department of Medicine, McMaster University, Hamilton, ON L8S 4K1, Canada ${ }^{30}$ School of Food Science and Technology, Jiangnan University, Wuxi,

${ }^{31}$ School of Food and Biological Engineering Hefei University of Technology, Hefei 230009, China

${ }^{32}$ Department of Analytical Chemistry and Food Science, University of Vigo, Vigo 36310, Spain

${ }^{33}$ Institute of Biochemistry and Molecular Biology, Northeastern University, Shenyang, Liaoning, 110819, P.R. China

${ }^{34}$ School of Chinese Medicine, Hong Kong Baptist University, Hong Kong, China ${ }^{35}$ Department of Biology, Selcuk University, Campus/Konya, Turkey

Correspondence Jesus Simal-Gandara, Department of Analytical Chemistry and Food Science, University of Vigo, Vigo 36310, Spain. Email: jsimal@uvigo.es 